\title{
Parietal Area VIP Causally Influences Heading Perception during Pursuit Eye Movements
}

\author{
Tao Zhang and Kenneth H. Britten \\ Center for Neuroscience, University of California, Davis, Davis, California 95618
}

The ventral intraparietal area (VIP) of the macaque monkey brain is a multimodal area with visual, vestibular, somatosensory, and eye movement-related responses. The visual responses are strongly directional, and VIP neurons respond well to complex optic flow patterns similar to those found during self-motion. To test the hypothesis that visual responses in VIP directly contribute to the perception of self-motion direction, we used electrical microstimulation to perturb activity in VIP while animals performed a two-alternative heading discrimination task. Microstimulation systematically biased monkeys' choices in a direction consistent with neuronal preferences at the stimulation site, and these effects were larger while the animal was making smooth pursuit eye movements. From these results, we conclude that VIP is causally involved in the perception of self-motion from visual cues and that this involvement is gated by ongoing motor behavior.

\section{Introduction}

All motile animals need to have a sense of where they are going. In monkeys and other terrestrial vertebrates, the dominant sense guiding locomotion is vision. In particular, complex patterns of motion (optic flow) provide an excellent source of information about the current heading direction (Gibson, 1950). The use of this cue has been extensively studied using perceptual, theoretical, and physiological approaches (for review, see Britten, 2008). However, the physiological mechanisms underlying this sense are only beginning to be understood. Relevant neuronal signals are found in a number of cortical areas, including the medial superior temporal area (MST) (Tanaka et al., 1986; Duffy and Wurtz, 1991; Graziano et al., 1994), the ventral intraparietal area (VIP) (Schaafsma and Duysens, 1996; Bremmer et al., 2002a; Zhang et al., 2004), area 7a (Siegel and Read, 1997), and even primary motor cortex (Merchant et al., 2001). Establishing how these multiple areas each contribute to the perception of heading is a difficult and important challenge for the field.

VIP is a multimodal area, with visual, auditory, somatosensory, vestibular, and eye movement-related responses (Colby et al., 1993; Duhamel et al., 1998; Bremmer et al., 2002b; Schlack et al., 2002, 2003, 2005). It has at least two unusual visual receptivefield (RF) properties. Many RFs in VIP are depth limited, such that effective stimuli will only drive the cell when presented at the correct range from the monkey; this range is usually nearer than

\section{Received Oct. 21, 2010; revised Dec. 6, 2010; accepted Dec. 16, 2010.}

This work was supported by the National Eye Institute Grant EY10562 (K.H.B.) and Vision Core Center Grant EY12576. We thank D. J. Sperka for software development and support, and H. R. Engelhardt for technical support and animal training. We are grateful to D. F. Cooke and S. W. Egger for providing thoughtful comments on a previous version of this paper.

T. Zhang's present address: State Key Laboratory of Brain and Cognitive Science, Institute of Psychology, Chinese Academy of Sciences, 4 A Datun Road, Chaoyang District, Beijing 100101, China.

Correspondence should be addressed to Kenneth H. Britten, Center for Neuroscience, University of California, Davis, 1544 Newton Court, Davis, CA 95618. E-mail: khbritten@ucdavis.edu.

DOI:10.1523/JNEUROSCI.5520-10.2011

Copyright $\odot 2011$ the authors $\quad 0270-6474 / 11 / 312569-07 \$ 15.00 / 0$ the fixation point (Colby et al., 1993). Second, many cells in this area compensate for the location of the eye in the orbit (Duhamel et al., 1997). In the extreme, this leads to an RF that encodes stimulus locations in head-centered coordinates; other cells show a range of coordinate systems from partially head centered to the more familiar eye centered. These properties suggest that VIP might play a role in encoding object locations in near-extrapersonal space.

To test the hypothesis that VIP directly contributes to heading perception, we stimulated selective sites in VIP while trained monkeys were performing a heading discrimination task. Neuronal signals for heading are clustered in VIP, which allows stimulation to activate a group of similarly tuned neurons near the electrode (Zhang and Britten, 2004). Monkeys' choices were systematically biased in favor of the stimulation site preference, supporting the hypothesis.

Smooth pursuit eye movements are an important part of primate behavior and form a particular challenge for any kind of motion perception. The eye movement creates a reafferent motion pattern on the retina, which adds to the motion pattern created by one's trajectory, greatly complicating the estimation of heading from optic flow. We have previously found that VIP neurons can compensate for the presence of eye movements (Zhang et al., 2004), so we included eye movement trials in the current microstimulation experiment. We found that the effects of microstimulation were larger and more systematic in the presence of smooth pursuit eye movements. This finding indicates that engagement of pursuit gates the perceptual effects of neuronal signals in parietal cortex.

\section{Materials and Methods}

Two adult female rhesus macaques were used in this study. Before recording, each was equipped with a head implant (including a head post and recording cylinder) and scleral search coil allowing us to hold the head still and record eye movements. These were implanted under deep anesthesia, using sterile techniques, in a dedicated animal surgery facility. 
Animals were trained on three tasks: fixation, delayed-saccade, and heading discrimination. Correct behavior was rewarded with a drop of juice, and mistakes were punished with a brief time-out period. Animals were maintained in an Association for Assessment and Accreditation of Laboratory Animal Care-certified vivarium, and all procedures were overseen by local veterinary staff. All procedures conformed to the Institute for Laboratory Animal Research Guide for the Care and Treatment of Laboratory Animals and were approved by the University of California, Davis, Institutional Animal Care and Use Committee.

Task and stimuli. The primary task from which the data in this paper derive is a two-alternative heading discrimination task, illustrated in Figure 1. In each trial, a single stimulus was presented, simulating approach at $1 \mathrm{~m} / \mathrm{s}$ toward a three-dimensional cloud of points, $10 \mathrm{~m}$ across. The display was presented by an ATI video card controlled by a dedicated display computer running real-time Linux, and the display refresh rate was $85 \mathrm{~Hz}$. Each point on the screen subtended $\sim 0.1^{\circ}$, and $\sim 2000$ points were in view. The luminance of the points was $60 \mathrm{~cd} / \mathrm{m}^{2}$, and the background luminance was dim room illumination, $\sim 0.1 \mathrm{~cd} / \mathrm{m}^{2}$. Individual $1 \mathrm{~s} \mathrm{stimuli}$ simulated linear trajectories with heading angles ranging from 0.5 to $8^{\circ}$ from directly ahead, on both sides, on $\log 2$ intervals. The monkey indicated its choice by making a saccade to one of two targets, presented after the stimulus disappeared. Monkeys were required to fixate in a $1-1.5^{\circ}$ window for the duration of the simulated trajectory. Correct trials were rewarded with a drop of juice.

Three eye movement conditions were randomly interleaved in a block of trials: fixation and horizontal pursuit at $10 \%$ s to the left and right. In a pursuit trial, the fixation target first stepped to a location opposite the pursuit direction, such that the midpoint of the target position sweep was the same as the fixation point location on a trial without pursuit.

Recording and stimulation. We recorded multiunit activity using platinum-iridium alloy electrodes coated with Parylene (FHC). Electrode signals were amplified (Bak Electronics), and multiunit activity was sorted with a window discriminator. Times of impulses were recorded by the experimental control computer (running REX; the National Institutes of Health-developed environment) with a resolution of $1 \mathrm{~ms}$. The intraparietal sulcus (IPS) was initially localized by magnetic resonance imaging, and electrodes were introduced into the IPS through a grid that supported the guide tubes according to $1 \mathrm{~mm}$ intervals. We began by mapping the IPS, identifying VIP by its characteristic physiological responses and by its depth in the sulcus. It was distinguished from the lateral intraparietal area (LIP) by having little or no sustained response during the delay period in a delayed-saccade task, and from the medial intraparietal area (MIP) by an absence of responses associated with spontaneous arm motions. Consistently robust and directional visual responses were the principal characteristics allowing us to be sure that the electrode was in VIP.

We recorded activity at frequent intervals along penetrations through VIP while continuously stimulating with the heading stimuli. To select sites for microstimulation, we identified regions showing consistently directional responses for a length of at least $500 \mu \mathrm{m}$ of electrode travel. The electrode was then moved to the midpoint of the site, and the multiunit receptive field was mapped by hand. The fixation point was adjusted to center the RF on the screen as well as possible, and the heading tuning was measured. Tuning data consisted of all headings from -30 to $30^{\circ}$ at $5^{\circ}$ intervals. Although a minority of single VIP neurons have been reported to possess RFs in headcentric or egocentric coordinates (Duhamel et al., 1997), we did not observe this phenomenon at the level of multiunit activity. We speculate that this is because such cells are randomly intermixed with cells possessing more conventional retinotopic receptive fields, or those with RFs intermediate between head- and eye-centered. In any case, all the data included in this paper came from experiments in which we could adequately center the RF on the screen. The electrode was then connected to a stimulus isolation unit (FHC), which delivered current pulses according to a pulse sequence controlled by a programmable pulse generator (A.M.P.I.). A complete block of trials consisted of 20 trials at each combination of 10 heading directions, three eye movement conditions, and two microstimulation conditions. After completion of the experiment, heading tuning was remeasured.

\section{a Simulated geometry}

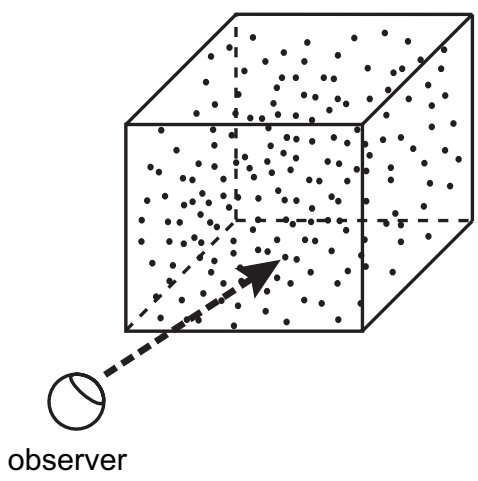

b Screen arrangement

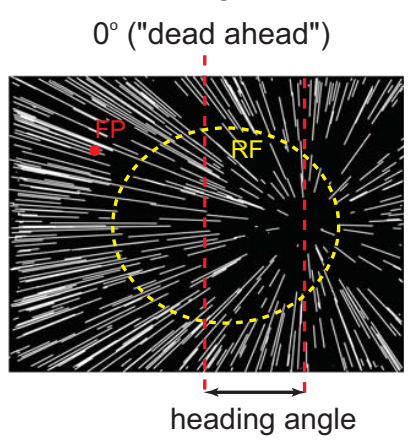

C Timing

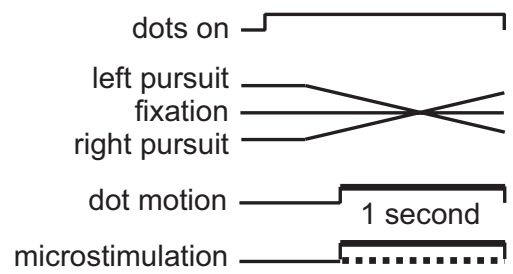

Figure 1. Schematic of visual stimuli and experimental procedures. $\boldsymbol{a}$, Geometry of simulated scene. $\boldsymbol{b}$, Observer view of the stimuli, showing the pattern of dot motion on the screen. The red dot is the fixation point, and the dashed yellow circle indicates the receptive field of a typical VIP stimulation site. The longer vertical dashed line depicts dead ahead. The heading angle is defined as the angle between dead ahead and the simulated trajectory. c, Timing of events in a trial.

Data analysis. Psychometric data from each eye movement and stimulation condition were separately fit with probit (cumulative Gaussian) functions as follows:

$$
P(r)=\left(\frac{1}{\sigma \sqrt{2 \pi}} \int_{-\infty}^{h} e^{\frac{-(x-\mu)^{2}}{2 \sigma^{2}}} d x\right) .
$$

These functions, which have been extensively used for this kind of data (Britten and van Wezel, 1998; Gu et al., 2007), provide a very good account of both the shape and position of the psychometric function. In this expression, $P(r)$ denotes the proportion of right choices; $\sigma$, the width of the function; and $\mu$, the midpoint, corresponding to the point of subjective dead ahead. The width parameter captures the sensitivity of the monkey to small changes of heading and corresponds to the amount of heading change from the midpoint that would produce $84 \%$ rightward choices.

To assess the effects of microstimulation, we compared both of these parameters from the two fits resulting from the control and stimulation data. Each data set was fit independently. To determine the statistical 


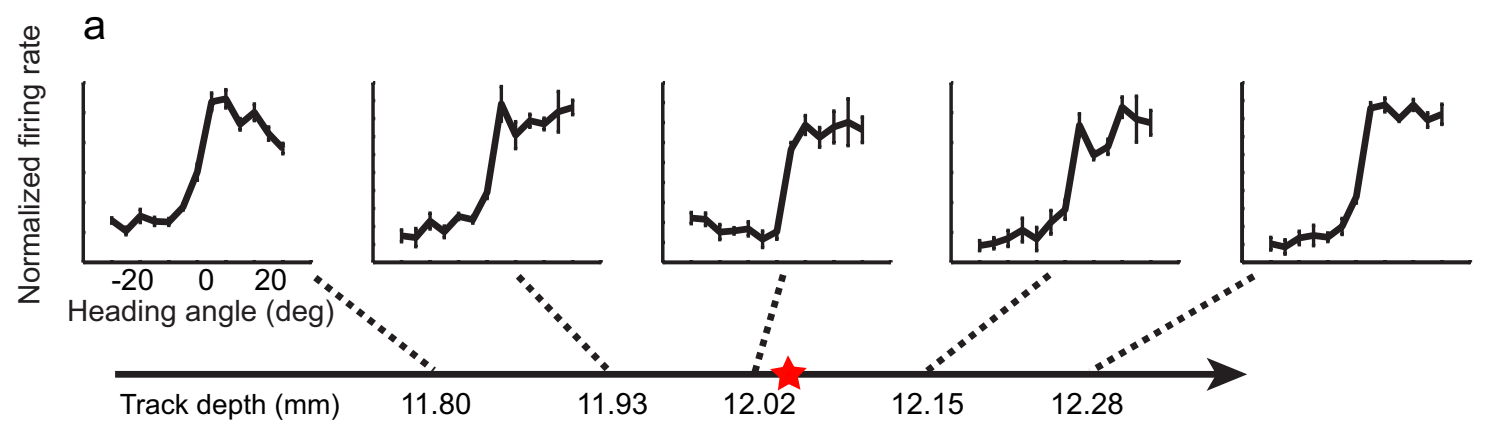

b

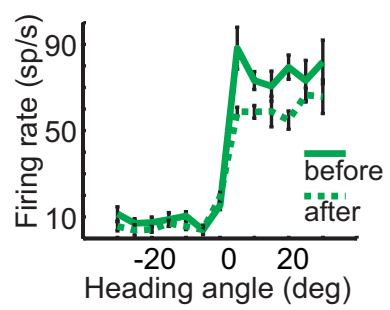

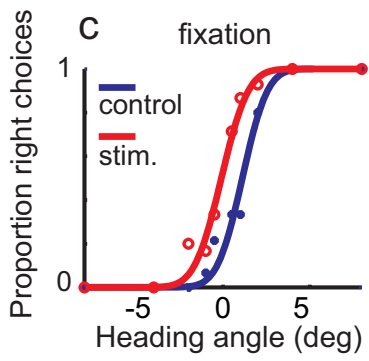

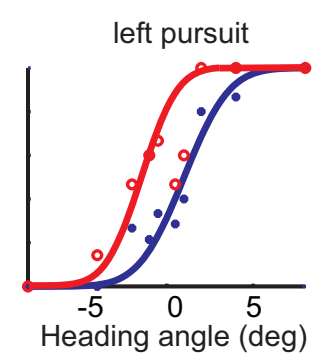

Figure 2. Example of one experiment showing microstimulation effects. The horizontal line with arrowhead represents the electrode penetration. Multiunit activity was sampled ( $\boldsymbol{a}$ ) along the path. Threshold for spike detection was set to achieve a maintained discharge from $20 \mathrm{to} 30 \mathrm{~Hz}$, and each recording was independently normalized to the maximum firing rate at that location. In this case, neurons with similar tuning properties were found on a $\sim 600 \mu \mathrm{m}$ distance along the penetration. Once a suitable $(>500 \mu \mathrm{m})$ cluster was found, we placed the tip of electrode at the center of the cluster, as indicated by the red star on the electrode trajectory. In this case, this location was in the putative deep layers, $\sim 500 \mu \mathrm{m}$ above the transition to white matter. Heading tuning curves before (solid green curve) and after (dashed green curve) microstimulation experiments are shown in $\boldsymbol{b}$. The tuning properties are well preserved. $\boldsymbol{c}$, Psychometric data resulting from this experiment. Midpoint shifts induced by microstimulation are $-1.28,-2.01$, and $-1.46^{\circ}$ (static, left, and right pursuit, respectively) and are consistent with the preference of this stimulation site. Error bars indicate SEM.

significance of the change in one of the parameters, the fit was repeated with a single free parameter for the midpoint or $\sigma$ for both data sets. The difference in $\chi^{2}$ values resulting from the two fits is also $\chi^{2}$ distributed and can be used to test the null hypothesis that the two functions were from the same distribution, with degrees of freedom corresponding to the difference in the number of free parameters in the two fits (Hoel et al., 1971).

To assess the degree of tuning of individual sites, we used a conventional contrast index $\left(R_{\text {right }}-R_{\text {left }}\right) /\left(R_{\text {right }}+R_{\text {left }}\right)$, which we termed the heading tuning index. This index was computed from the average across nonzero headings in the tuning data set.

\section{Results}

We tested the involvement of VIP in heading perception in two adult female rhesus macaques (Macaca mulatta). Before the experiment began, each was fully trained on a two-alternative heading discrimination task that we have used in previous experiments (Britten and van Wezel, 1998, 2002; Zhang and Britten, 2010). The task and stimuli are illustrated in Figure 1. The stimuli simulate a $1 \mathrm{~s}$ trajectory toward a three-dimensional cloud of points, which can either be to the left or right of directly ahead. The monkey reports its decision by making a saccadic eye movement to the corresponding saccade target, which is presented after the end of the stimulus period. We used a range of different heading angles, allowing us to measure a psychometric function relating choice to heading direction. Three different eye movement conditions were randomly interleaved: fixation and two directions of horizontal pursuit at $10 \%$ s.

VIP was identified by its anatomical location and its distinctive responses (for details, see Materials and Methods). We identified VIP microstimulation sites by systematically mapping multiunit heading tuning along penetrations through VIP. Sites were accepted if they maintained a consistent preference for left or right headings for $>500 \mu \mathrm{m}$. An example of such a site is shown in Figure $2 a$; this site preferred rightward headings. The electrode was then positioned in the middle of the site and connected to the stimulus isolator for microstimulation.

On 50\% of trials in each microstimulation experiment, a train of current pulses were delivered through the electrode $(200 \mathrm{~Hz}$, $40 \mu \mathrm{A}$ peak-to-peak, biphasic, $100 \mu$ s pulse duration, with $75 \mu \mathrm{s}$ intervening between phases). The microstimulation trials were randomly interleaved with control trials, and all conditions were equally represented in a block of trials. In this experiment, the psychometric functions resulting from the stimulated trials were systematically shifted upward, corresponding to a bias in favor of rightward headings (Fig. 2c). After the experiment, we remeasured the heading tuning to confirm that the electrode had not shifted and that the region surrounding the electrode was still responsive. The results from this confirmation are shown in Figure $2 b$. This experiment demonstrates that restricted stimulation of VIP can produce substantial biases in the perception of self-motion, concordant with the heading preference of the region being stimulated.

We quantified this perceptual effect by estimating the horizontal shift between the stimulated and control data. Each was fit with a probit function; the difference between their midpoints expresses the perceptual effect in units of the stimulus. All three of these functions were significantly shifted (bootstrap test, $p<$ 0.05). To produce a single estimate of the shift for each site, we averaged the effects for all three pursuit conditions. We also expressed the resulting shift relative to the heading preference of the stimulation site. The results of 60 experiments are shown in Figure $3 a$. The average of this distribution is shifted to the right, consistent with site preference, but this shift is only marginally significant ( $t$ test, $p=0.07$ ). The majority of cases that were individually significant by the bootstrap test (filled bars) were also shifted to the right (also see Table 1). 

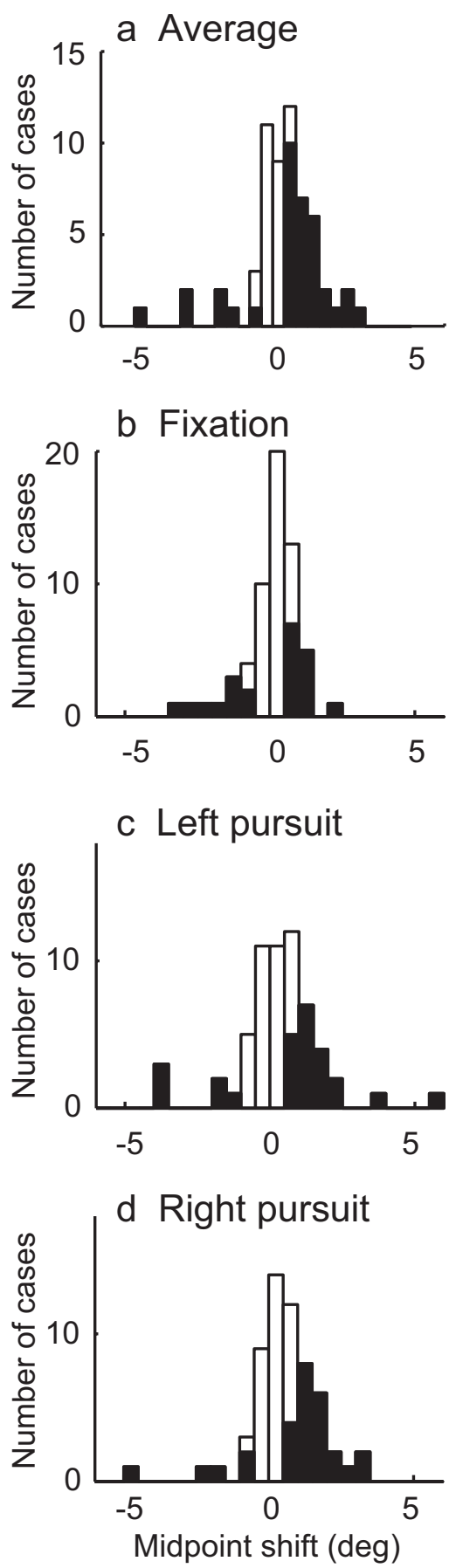

Figure 3. Sample summary of stimulation effects on choice. For each case, we assessed whether the shift was in the direction of the site preference (positive values) or opposed. $\boldsymbol{a}$, The average shift across pursuit conditions was moderately positive ( $t$ test, $p=0.07$ ). $\boldsymbol{b}-\boldsymbol{d}$, Individual pursuit conditions. The shifts were much larger for the two pursuit conditions than for the fixation conditions (for statistical results, see Table 1). The filled bars denote statistically significant cases (bootstrap test, $p<0.05$ ).

The modest effects of stimulation on perception became much more evident when we separated the data by eye movement condition. Without pursuit, no systematic effect of stimulation on decision was evident, although a minority of individual cases was shifted in either direction (Fig. 3b). However, with either direction of pursuit, the effects of microstimulation were larger and more systematically aligned with the preference of the site (Fig. 3c,d; Table 1).
The dependence of the magnitude of the microstimulation effects on eye movements is most evident in scatterplot form (Fig. 4 ). This figure shows the magnitude of the stimulation effect on fixation trials ( $x$-axis) against the effect on pursuit trials (both directions combined). In the majority of cases, the effects were larger during pursuit, and this difference was highly significant (paired $t$ test, $p<0.001$ ). The effects of microstimulation were increased similarly for both directions of pursuit (indicated by the colors in Fig. 4). However, there was no systematic relationship between the presence of pursuit and stimulation-induced changes in the slope of the psychometric function (see Fig. 6). We confirmed that there was no interaction between the main results and any stimulation effects on slope by reexpressing the shifts relative to the slope, and the results of this analysis are shown in supplemental Figure 1 (available at www.jneurosci.org as supplemental material).

To confirm the effect of stimulation on behavior, we performed a repeated-measures ANOVA, with the stimulationinduced shift as the dependent variable, and with monkey and pursuit condition as independent variables. The main effect of stimulation was significant $(p<0.001)$, as was the interaction with pursuit $(p=0.015)$. There was no significant difference between monkeys ( $p=0.62$ ).

Many studies using microstimulation have reported that the sites carrying the most substantial or sensitive signals regarding the stimuli also exerted the greatest leverage on perception (Salzman et al., 1990; Britten and van Wezel, 1998; DeAngelis et al., 1998; Uka and DeAngelis, 2006). This is taken as strong evidence supporting a causal role for the signals in perception: higher amplitude signals are favored in the readout of sensory representations. This hypothesis is of particular interest in a complex, multimodal representation like that found in VIP, since multiple dimensions complicate the readout. To examine this question, we related the microstimulation-induced shifts to a contrast index capturing the selectivity of the heading signals at individual sites (Fig. 5). This index will have a value of -1 for a completely left-preferring site and 1 for a right-preferring site. The results are very consistent with an increased impact of microstimulationand presumably of endogenous VIP signals-during pursuit. There was a weak relationship between heading selectivity during fixation and the effect of microstimulation (Fig. $5 a$ ), which became somewhat stronger during pursuit in either direction (Fig. 5b,c). Cases with low index values arose from two distinct causes, low slopes and non-monotonic tuning (either peaks or troughs near central headings). The majority of our case arose from the former cause, but it is interesting that non-monotonically tuned sites also yielded weak effects on perception, consistent with what one might expect on theoretical grounds.

Although we were most interested in biases induced by stimulation, we also examined changes in sensitivity (Fig. 6). Reduction of sensitivity might be expected if the microstimulation were injecting noise into cortical heading signals, and increases might be expected if something akin to attention were activated. In our analysis, changes of sensitivity manifest themselves as changes in the slopes of the psychometric functions, with steeper slope indicating higher sensitivity. Only a minority of cases showed individually significant effects on slope, and these were not systematically in one direction or the other. A repeated-measures ANOVA also showed no consistent slope shift for the 60 experiments, nor any dependence on pursuit or monkey (all factors, $p>0.25$ ). From this, we conclude that microstimulation exerts its main effect on the net balance of cortical signals in favor of each of the two alternatives, but not on the overall efficiency of processing of heading information. 
Table 1. Statistical results

\begin{tabular}{|c|c|c|c|c|c|c|}
\hline \multirow[b]{2}{*}{$N=60$} & \multicolumn{2}{|l|}{ Fixation } & \multicolumn{2}{|l|}{ Left pursuit } & \multicolumn{2}{|l|}{ Right pursuit } \\
\hline & Midpoint shifts & Slope changes & Midpoint shifts & Slope changes & Midpoint shifts & Slope changes \\
\hline Mean (deg) & 0.06 & 0.11 & 0.32 & 0.07 & 0.55 & 0.14 \\
\hline$p(t$ test $)$ & 0.63 & 0.20 & 0.10 & 0.72 & $<0.01$ & 0.25 \\
\hline Significant cases: preferred/total & $13 / 22(59 \%)$ & $5 / 6(83 \%)$ & $20 / 26(77 \%)$ & $3 / 9(33 \%)$ & $23 / 28(82 \%)$ & $6 / 8(75 \%)$ \\
\hline
\end{tabular}

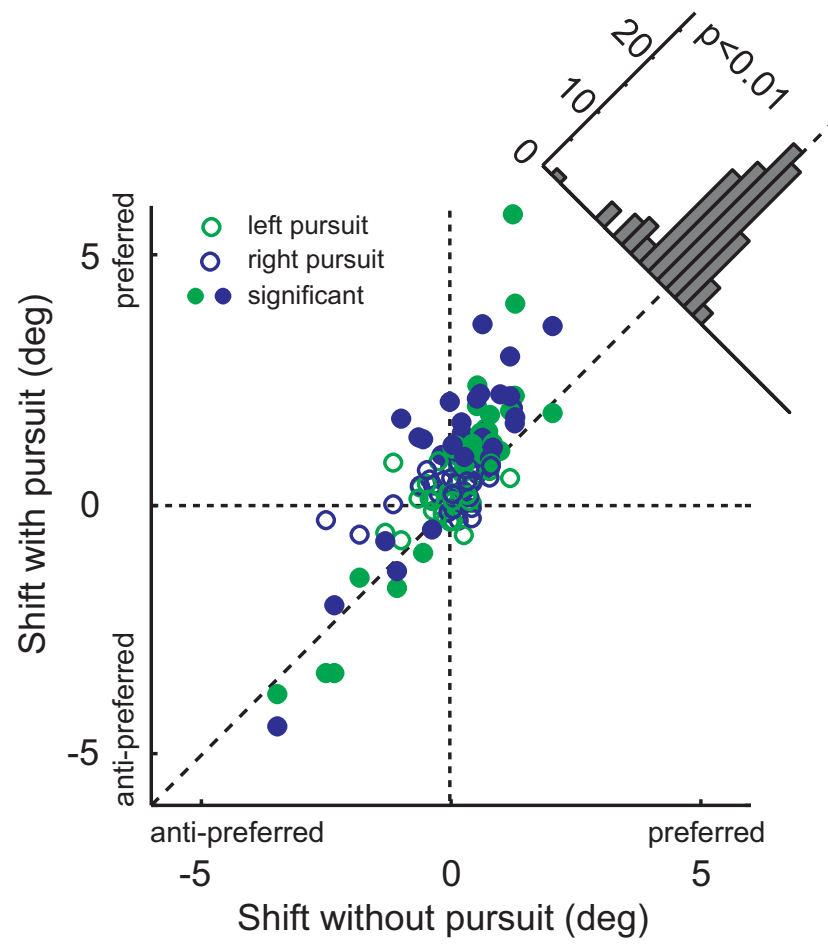

Figure 4. Interaction between pursuit and microstimulation effects. We plotted midpoint shift with pursuit against shift under fixation. Points are systematically shifted above the diagonal line that indicates equal effect. This shift is statistically significant (paired $t$ test, $p<$ 0.001). Significant shifts for the pursuit condition are indicated by filled symbols.

\section{Discussion}

VIP is a complex, multimodal area carrying numerous sensory signals. As such, it is likely to be involved in multiple sensory-guided behaviors. One behavior for which it seems well suited is navigation (Bremmer, 2005). We tested the hypothesis that VIP is directly and causally involved in discrimination of heading based on visual cues. We found that stimulation of VIP biases heading judgments and does so more strongly while the monkey is engaged in pursuit eye movements. These results support the hypothesis and suggest that the behavioral significance of neuronal signals in VIP depends on the context in which the discrimination is made.

\section{Relationship with previous work}

VIP has many properties that make it well suited for a role in the guidance of self-motion. It is located in the parietal cortex, which is implicated in spatial guidance of movement (Snyder et al., 2000), as well as in the control of spatial attention (Bisley and Goldberg, 2010). VIP receives dense feedforward input from MT (Maunsell and Van Essen, 1983), which places it in a position in the dorsal motion pathway comparable with the medial superior temporal area (Felleman and Van Essen, 1991).

VIP has a constellation of physiological properties that make it unique in cortex. Although more of its cells respond to visual stimulation than to any other modality, it also has cells respond-
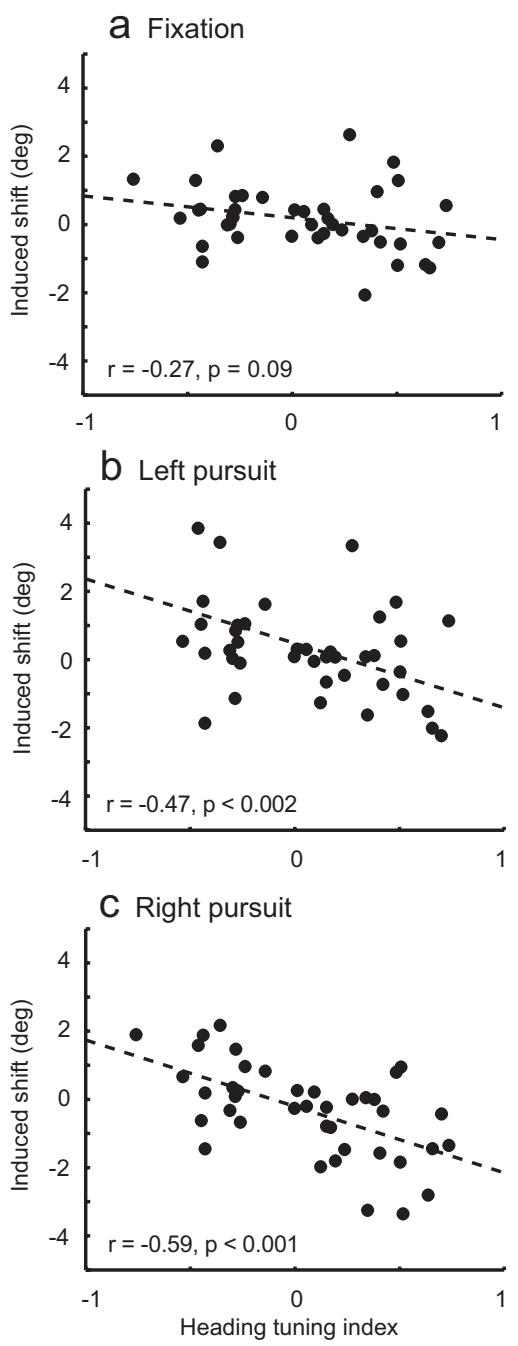

Figure 5. $\boldsymbol{a}-\boldsymbol{c}$, Relationship between stimulation effect and site tuning strength for each fixation condition. The difference in slopes was only marginally significant, statistically (ANCOVA, $p=0.083$ ). This analysis only included the 41 sites for which we collected tuning data.

ing to auditory (Schlack et al., 2005), somatosensory (Duhamel et al., 1998), and vestibular (Schlack et al., 2002) stimulation. Auditory and visual receptive fields tend to be spatially circumscribed, and also frequently are circumscribed with respect to depth, even when monocularly viewed (Colby et al., 1993).

Most VIP neurons are strongly directional (Colby et al., 1993) and are selective for complex motion patterns in a manner very reminiscent of MST (Schaafsma and Duysens, 1996). We have shown that these areas are quantitatively as well as qualitatively similar when studied under identical conditions (Maciokas and Britten, 2010). These observations led us to investigate VIP in the context of our heading task. We have previously shown that the responses of VIP neurons are remarkably stable in the face of pursuit, as is perception (Zhang et al., 2004), and that the most sensitive 


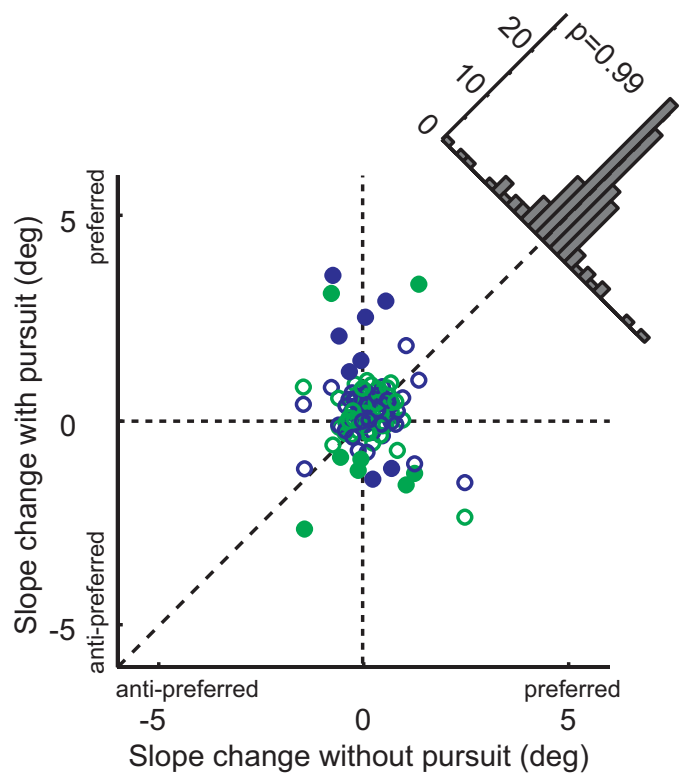

Figure 6. Effects of microstimulation on the slope of the psychometric function. Conventions are as in Figure 4.

neurons in VIP are sufficient to support perceptual levels of sensitivity to small heading changes (Zhang and Britten, 2010).

These observations make a circumstantial case that VIP is involved in heading discrimination. The present observations allow a much stronger conclusion: VIP has a causal role in heading discrimination. Its responses are quite similar to those of MST, when perturbed in the same way (Britten and van Wezel, 1998, 2002). Thus, the two areas, which differ in many other important ways, nonetheless contribute approximately equivalently to heading perception. The only other comparable pair of observations involves microstimulation of areas MT and MST in the context of a linear direction discrimination task. These two areas also bias monkey judgments rather similarly (Salzman et al., 1992; Celebrini and Newsome, 1994). However, this result is much more expected than the present results, given the many similarities between the directional signals in areas MT and MST. The present results make a stronger case that two areas with many dissimilar and some similar properties can contribute equally to a task that taps into their similarities. This finding supports a view in which signals appropriate to a task, wherever they might be found in cortex, can be recruited to improve performance.

The one previous microstimulation experiment in VIP differed in important technical ways from our experiment. Cooke et al. (2003) stimulated VIP in monkeys that were alert yet not engaged in any task. The result was stereotyped movements of the face, head, and shoulder, closely resembling natural movements to avoid unpleasant stimuli (Cooke and Graziano, 2003). We closely observed our monkeys in the present experiments using closed-circuit television and never observed such movements. Several differences between the experiments might contribute to this. First, the threshold currents to evoke movement were, on average, $\sim 4.5$ times as large as were ours. In addition, the pulse duration in the Cooke experiments was $400 \mu$ s, instead of the 100 $\mu$ s we used. Therefore, vastly greater charge transfer occurred, and very likely much greater volumes of cortex were activated (Asanuma, 1981). Additionally, in the Cooke experiments, stimulation sites were chosen at random, whereas ours were carefully selected based on their visual response properties. This aspect, however, seems unlikely to have been critical, since $>95 \%$ of sites produced movements, and our selection criteria certainly did not exclude such a large fraction of sites. Last, in the Cooke experiments, the monkeys were not actively engaged in a task, which might greatly affect the outcome. Indeed, in our experiments, the seemingly minor variation of being engaged in pursuit substantially influenced the results of stimulation (see below). Despite these differences in procedures, we find it intriguing that activation of a single cortical region can have such divergent behavioral outcomes. We believe this pair of results, together, falsifies any hypothesis suggesting that individual cortical areas are specialized for a single behavioral role.

\section{Coordinate frames and eye movements}

There is a large literature on the coordinate transforms evident in both sensory and motor activity in parietal cortical areas (for review, see Buneo and Andersen, 2006). Although much of the interest lies in the coordination of eye and hand movements, related issues arise in the guidance of locomotor behavior. Two visual cues (in addition to other modalities) are thought to drive locomotor movements: the positions of targets or obstacles in extrapersonal space, and optic flow signals informative about the current trajectory (Fajen and Warren, 2003). Both of these cues are highly affected by reafference from the movements of the eyes. Obviously, the positions of objects-like those that are the targets of reaching movements-must be represented in a coordinate frame useful for locomotor behavior. VIP is interesting because the RFs of some neurons are represented in a head-centered coordinate frame (Duhamel et al., 1997), or in frames intermediate between eye and head centered (Avillac et al., 2005). This range of coordinate systems allows for more flexible computation (Ben Hamed et al., 2003). This idea is consistent with the view that VIP is used in a variety of tasks; having a flexible representation is probably necessary for multiple behavioral roles.

The problem of taking into account the movements of the eyes is particularly severe for computations based on optic flow. Smooth pursuit eye movements distort the retinal flow field, making it more difficult to recover the direction of heading (Royden et al., 1992). VIP neurons show a range of different coordinate systems with respect to eye velocity, as well as eye position, when representing heading direction (Zhang et al., 2004). This is provocative with respect to the present work, since the effects of microstimulation were larger under pursuit than when the eye was stationary. Although this relationship was quite consistent across stimulation sites, we can draw no strong conclusions because we do not know at present whether pursuit stability is clustered anatomically nor the exact dimensions of the area activated by microstimulation. However, we speculate that the effects of microstimulation depend on pursuit simply because heading perception is much easier while fixating - one merely needs to identify the focus of expansion in the image to solve the problem. There are many more cortical areas with information useful for this simpler problem, but only a few areas have been identified with appropriate properties to discriminate heading under pursuit. Therefore, the change in the relationship of VIP to behavior represents active rearrangement of which cortical signals are useful on a given trial. Where and how this gating occurs is a fascinating, unsolved problem.

\section{The many roles of VIP}

We believe the present results, together with previous evidence, argue against VIP being highly specialized for any single function like navigation or object avoidance. Instead, it is likely that signals 
in VIP support many different functions. Although laboratory experiments tend to isolate particular behavioral tasks for study, in natural circumstances many of the same behaviors are performed simultaneously. For instance, object avoidance is a natural part of movement through dense habitat, and eye movements are systematically related to one's trajectory and to features in the scene (Land and Lee, 1994; Niemann et al., 1999). It is interesting that the different motor-related regions of the intraparietal sulcus (MIP and LIP) appear to have strong preferences for single effectors, even when studied in tasks in which both eye and limb movements are involved (Buneo and Andersen, 2006). The location of VIP, and its dense connections to other more modalityspecific regions, has led anatomists to propose that it might have a major integrative role (Jones and Powell, 1970; Seltzer and Pandya, 1980; Lewis and Van Essen, 2000). Our microstimulation data provide strong support for this hypothesis. To understand how the different regions of parietal cortex work together in natural behavior, it might be necessary to perform more complex experiments with multiple stimuli and effectors.

\section{References}

Asanuma H (1981) Microstimulation technique. In: Electrical stimulation research techniques (Patterson MM, Kesner RP, eds), pp 61-70. New York: Academic.

Avillac M, Denève S, Olivier E, Pouget A, Duhamel JR (2005) Reference frames for representing visual and tactile locations in parietal cortex. Nat Neurosci 8:941-949.

Ben Hamed S, Page W, Duffy C, Pouget A (2003) MSTd neuronal basis functions for the population encoding of heading direction. J Neurophysiol 90:549-558.

Bisley JW, Goldberg ME (2010) Attention, intention, and priority in the parietal lobe. Annu Rev Neurosci 33:1-21.

Bremmer F (2005) Navigation in space - the role of the macaque ventral intraparietal area. J Physiol 566:29-35.

Bremmer F, Duhamel JR, Ben Hamed S, GrafW (2002a) Heading encoding in the macaque ventral intraparietal area (VIP). Eur J Neurosci 16:1554-1568.

Bremmer F, Klam F, Duhamel JR, Ben Hamed S, Graf W (2002b) Visualvestibular interactive responses in the macaque ventral intraparietal area (VIP). Eur J Neurosci 16:1569-1586.

Britten KH (2008) Mechanisms of self-motion perception. Annu Rev Neurosci 31:389-410.

Britten KH, van Wezel RJ (1998) Electrical microstimulation of cortical area MST biases heading perception in monkeys. Nat Neurosci 1:59-63.

Britten KH, van Wezel RJ (2002) Area MST and heading perception in macaque monkeys. Cereb Cortex 12:692-701.

Buneo CA, Andersen RA (2006) The posterior parietal cortex: sensorimotor interface for the planning and online control of visually guided movements. Neuropsychologia 44:2594-2606.

Celebrini S, Newsome WT (1994) Microstimulation of extrastriate area MST influences perceptual judgements of motion direction. Invest Ophthal Vis Sci 35:1828.

Colby CL, Duhamel JR, Goldberg ME (1993) Ventral intraparietal area of the macaque: anatomic location and visual response properties. J Neurophysiol 69:902-914

Cooke DF, Graziano MS (2003) Defensive movements evoked by air puff in monkeys. J Neurophysiol 90:3317-3329.

Cooke DF, Taylor CS, Moore T, Graziano MS (2003) Complex movements evoked by microstimulation of the ventral intraparietal area. Proc Natl Acad Sci U S A 100:6163-6168.

DeAngelis GC, Cumming BG, Newsome WT (1998) Cortical area MT and the perception of stereoscopic depth. Nature 394:677-680.

Duffy CJ, Wurtz RH (1991) Sensitivity of MST neurons to optic flow stimuli. I. A continuum of response selectivity to large-field stimuli. J Neurophysiol 65:1329-1345.

Duhamel JR, Bremmer F, BenHamed S, Graf W (1997) Spatial invariance of visual receptive fields in parietal cortex neurons. Nature 389:845-848.

Duhamel JR, Colby CL, Goldberg ME (1998) Ventral intraparietal area of the macaque: congruent visual and somatic response properties. J Neurophysiol 79:126-136.

Fajen BR, Warren WH (2003) Behavioral dynamics of steering, obstacle avoidance, and route selection. J Exp Psychol Hum Percept Perform 29:343-362.

Felleman DJ, Van Essen DC (1991) Distributed hierarchical processing in the primate cerebral cortex. Cereb Cortex 1:1-47.

Gibson JJ (1950) Perception of the visual world. Boston: Houghton-Mifflin.

Graziano MS, Andersen RA, Snowden RJ (1994) Tuning of MST neurons to spiral motions. J Neurosci 14:54-67.

Gu Y, DeAngelis GC, Angelaki DE (2007) A functional link between area MSTd and heading perception based on vestibular signals. Nat Neurosci 10:1038-1047.

Hoel P, Port S, Stone C (1971) Introduction to statistical theory. Boston: Houghton Mifflin.

Jones EG, Powell TP (1970) An anatomical study of converging sensory pathways within the cerebral cortex of the monkey. Brain 93:793-820.

Land MF, Lee DN (1994) Where we look when we steer. Nature 369:742-744.

Lewis JW, Van Essen DC (2000) Corticocortical connections of visual, sensorimotor, and multimodal processing areas in the parietal lobe of the macaque monkey. J Comp Neurol 428:112-137.

Maciokas JB, Britten KH (2010) Extrastriate area MST and parietal area VIP similarly represent forward headings. J Neurophysiol 104:239-247.

Maunsell JH, Van Essen DC (1983) The connections of the middle temporal visual area (MT) and their relationship to a cortical hierarchy in the macaque monkey. J Neurosci 3:2563-2586.

Merchant H, Battaglia-Mayer A, Georgopoulos AP (2001) Effects of optic flow in motor cortex and area 7a. J Neurophysiol 86:1937-1954.

Niemann T, Lappe M, Büscher A, Hoffmann KP (1999) Ocular responses to radial optic flow and single accelerated targets in humans. Vision Res 39:1359-1371.

Royden CS, Banks MS, Crowell JA (1992) The perception of heading during eye movements. Nature 360:583-585.

Salzman CD, Britten KH, Newsome WT (1990) Cortical microstimulation influences perceptual judgements of motion direction. Nature 346:174-177.

Salzman CD, Murasugi CM, Britten KH, Newsome WT (1992) Microstimulation in visual area MT: effects on direction discrimination performance. J Neurosci 12:2331-2355.

Schaafsma SJ, Duysens J (1996) Neurons in the ventral intraparietal area of awake macaque monkey closely resemble neurons in the dorsal part of the medial superior temporal area in their responses to optic flow patterns. J Neurophysiol 76:4056-4068.

Schlack A, Hoffmann KP, Bremmer F (2002) Interaction of linear vestibular and visual stimulation in the macaque ventral intraparietal area (VIP). Eur J Neurosci 16:1877-1886.

Schlack A, Hoffmann KP, Bremmer F (2003) Selectivity of macaque ventral intraparietal area (area VIP) for smooth pursuit eye movements. J Physiol 551:551-561.

Schlack A, Sterbing-D’Angelo SJ, Hartung K, Hoffmann KP, Bremmer F (2005) Multisensory space representations in the macaque ventral intraparietal area. J Neurosci 25:4616-4625.

Seltzer B, Pandya DN (1980) Converging visual and somatic sensory cortical input to the intraparietal sulcus of the rhesus monkey. Brain Res 192:339-351.

Siegel RM, Read HL (1997) Analysis of optic flow in the monkey parietal area 7a. Cereb Cortex 7:327-346.

Snyder LH, Batista AP, Andersen RA (2000) Intention-related activity in the posterior parietal cortex: a review. Vision Res 40:1433-1441.

Tanaka K, Hikosaka K, Saito H, Yukie M, Fukada Y, Iwai E (1986) Analysis of local and wide-field movements in the superior temporal visual areas of the macaque monkey. J Neurosci 6:134-144.

Uka T, DeAngelis GC (2006) Linking neural representation to function in stereoscopic depth perception: roles of the middle temporal area in coarse versus fine disparity discrimination. J Neurosci 26:6791-6802.

Zhang T, Britten KH (2004) Clustering of selectivity for optic flow in the ventral intraparietal area. Neuroreport 15:1941-1945.

Zhang T, Britten KH (2010) The responses of VIP neurons are sufficiently sensitive to support heading judgments. J Neurophysiol 103:1865-1873.

Zhang T, Heuer HW, Britten KH (2004) Parietal area VIP neuronal responses to heading stimuli are encoded in head-centered coordinates. Neuron 42:993-1001. 